\title{
AN ELECTROSTATIC BEAM LINE FOR ACCELERATOR MASS SPECTROSCOPY OF EXOTIC PARTICLES
}

\author{
D. ELMORE, P.W. KUBIK, T. HEMMICK and R. TENG \\ Nuclear Structure Research Laboratory, University of Rochester, Rochester, NY 14627, USA
}

H. KAGAN, P. HAAS, R.N. BOYD and R. TURNER

Department of Physics, Ohio State University, Columbus, OH 43210, USA

D. NITZ and D. CIAMPA

Department of Physics, University of Michigan, Ann Arbor, MI 48109, USA

\author{
S.L. OLSEN, T. GENTILE and T. HAELEN \\ Department of Physics. University of Rochester, Rochester, NY 14627, USA
}

\begin{abstract}
An all-electrostatic charged particle spectrometer has been constructed to perform high sensitivity searches for exotic states of matter. This spectrometer consists of an electrostatic beam line capable of mass independent charged particle transport and selection together with time-of-flight, energy loss and total energy detectors. This system has been used in conjunction with the tandem electrostatic accelerator at the Nuclear Structure Research Laboratory of the University of Rochester to search for fractionally charged or anomalously heavy particles.
\end{abstract}

\section{Introduction}

At present two notions are central to our understanding of the elementary processes of nature: the existence of elementary fractionally charged particles and of some mechanism to generate a mass scale. Our current understanding of the basic structure of matter has, as a central feature, the idea that hadronic matter is made from fractionally charged constituent particles called quarks. The experimental evidence upon which this theory is based is broad and persuasive (see, e.g., the review in ref. [1]). The known hadrons are presumed to be made up of those combinations of quarks which yield zero or integral values of electric charge. Whether or not free fractionally charged particles can exist in nature is a question of fundamental significance. The conservation of electric charge implies that a fractionally charged state would be stable. Thus the most sensitive searches involve lonking for fractionally charged components of terrestrial matter. These would exist in nature as remnants of the "big bang" or as a result of production in ultra-high energy cosmic ray interactions. So far, with one notable exception [2], all experimental efforts to confirm the existence of these exotic forms of matter have failed (references to previous work can be found in ref. [3]).

In the 1960s, Glashow, Weinberg and Salam [4] developed a model which unified the weak and electromagnetic interactions. The primary feature of this model is the existence of massive gauge bosons, the $W^{ \pm}$and $Z^{0}$. However, the masses of these bosons, which determine the relative strength of the weak and electromagnetic interactions, are unspecified by the theory. This question has been addressed by Technicolor models [5] which predict heavy (1-2 $\times 10^{3}$ amu) stable (halflife $-10^{16}$ years) particles of zero and integral charge. A heavy charge $=1+$ particle would appear in nature as a very heavy isotope of hydrogen. Smith and Bennett [6] have carried out extremely sensitive searches for hydrogen isotopes up to masses of $1200 \mathrm{amu}$ and found none at a level of 1 part in $10^{30}$. Negatively charged heavy particles bound electrostatically to a nucleus of atomic number $Z$ would act chemically as a heavy isotope of the element with atomic number $Z-1$. Middleton et al. [7] have ruled out isotopes of beryllium with masses up to $93 \mathrm{amu}$ at a level of about 1 part in $10^{12}$ nucleons and isotopes of oxygen up to a mass of 54 amu at a level of 1 part in $10^{18}$ nucleons. Dick et al. [8] have ruled out heavy isotopes of sodium for masses above $100 \mathrm{amu}$ at a level of 1 part in $10^{11}$ nucleons.

During the past two years we have designed and constructed a mass independent, all-electrostatic charged particle spectrometer capable of performing high sensilivity searches for exotic particles of unknown mass. 
Our philosophy has been that these particles, if they exist, may be hidden by their anomalous chemical behavior or heavy mass, for example in the residue of distillation processes. Hence we have attempted to build a system which has few restrictions on the physical or chemical form of the samples.

\section{The all-electrostatic spectrometer}

Figs. 1 and 2 show an overall view of the all-electrostatic charged particle spectrometer constructed at the Nuclear Structure Research Laboratory. A cesium sputter source [9] feeds directly into the tandem electrostatic accelerator through two electrostatic lenses. This source uses a primary cesium beam to sputter the sample and form negative ions. There are few restrictions on the physical or chemical form of the samples; atomic or molecular negative ion beams can be formed with all elements except some noble gases. Gas and solid samples have been used. Efficiencies for negative ion extraction from the source range from $0.1 \%$ to $1 \%$ depending on the physical and chemical composition of the sample. The total current is limited to about $2 \mu \mathrm{A}$ since higher currents tend to load down the accelerator. Compounds containing large amounts of oxygen, sulfur and halogens are avoided since these elements produce beams of many microamperes. Typical currents for atomic beams are $10 \mathrm{nA}$ for beryllium, $100 \mathrm{nA}$ for boron and 1 $\mu \mathrm{A}$ for amorphous carbon.

Following the accelerator, particles are focused with an electrostatic quadrupole doublet located between the last acceleration tube and the exit of the accelerator pressure vessel. After the quadrupole doublet, but still within the pressure vessel, a pair of electrostatic plates deflect the beam $1.3^{\circ}$ off axis to a set of tantalum slits and a removable Faraday cup. The beam then passes through a removable foil stripper, which is used to change the charge states of the ions before they reach the primary analyzer. Following the work of Dmitriev and Nikolaev [10] we have estimated the stripping efficiency for beryllium in a $5 \mu \mathrm{g} / \mathrm{cm}^{2}$ carbon foil. Roughly $0.02 \%$ of $10 \mathrm{MeV}^{9} \mathrm{Be}$ ions strip to a $1+$ charge state.

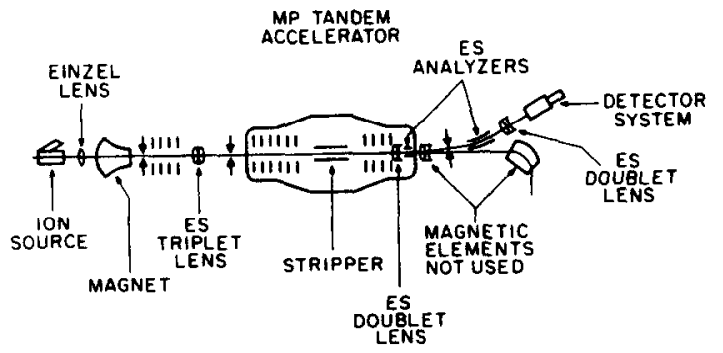

Fig. 1. A plan view of the apparatus including the injection system into the accelerator.

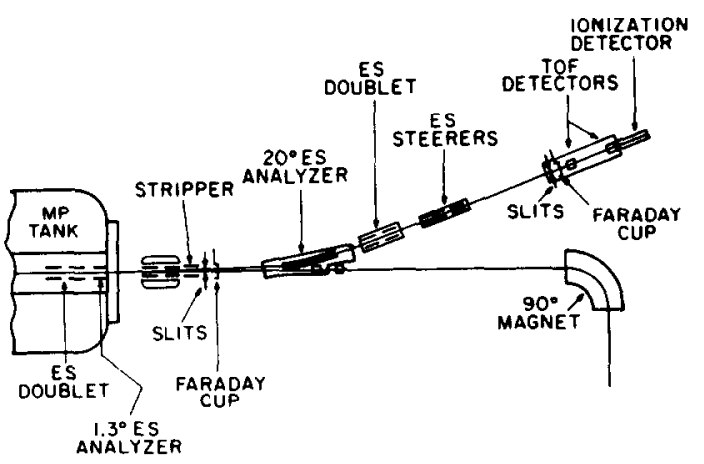

Fig. 2. A plan view of the high energy transport and detection system. The second foil stripper is situated at the focus of the quadrupole doublet which is located inside the accelerator's pressure tank.

We estimate that a 1000 amu $\mathrm{Be}$ isotope will strip to a $1+$ charge state $20 \%$ of the time. A foil stripper thus enhances the heavy mass signal by about a factor of $10^{3}$.

The $20^{\circ}$ high resolution analyzer is located $7.5 \mathrm{~cm}$ off the undeflected beam axis. It consists of solid aluminum electrodes with a $2.5 \mathrm{~cm}$ gap and a $500 \mathrm{~cm}$ radius of curvature. This analyzer selects particles with a specified ratio of kinetic energy to charge with a resolving power of $500 \mathrm{fwhm}$. Particles transmitted by the $20^{\circ}$ analyzer are studied in the detection system. In order to minimize backgrounds due to charge exchange or pick-up reactions the pressure in the analyzer is kept below $2 \times 10^{-8}$ Torr. The analyzer is followed by an electrostatic quadrupole doublet and a set of electrostatic steerers which focus particles emerging from the analyzer onto a combined time-of-flight and gas ionization detector. An aperture ladder and Faraday cup in front of the detector are used during beam tuning.

Fig. 3 shows a side view of the detector. The timeof-flight system consists of two detectors $1 \mathrm{~m}$ apart. Each detector consists of dual microchannel plates [11] which detect the electrons produced when beam par-

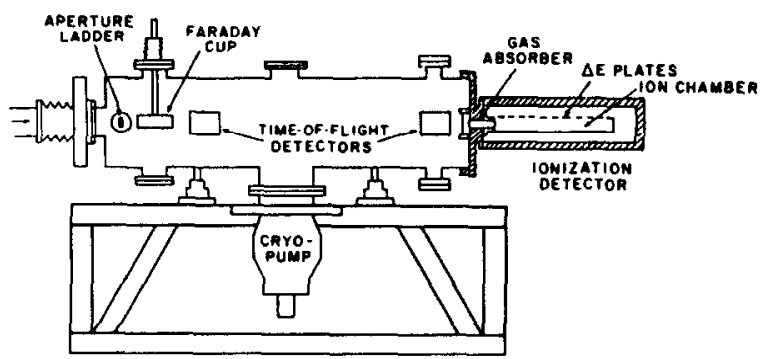

Fig. 3. A side view of the detection system. Two time-of-flight detectors are situated in the vacuum chamber. A gas cell separates the gas ionization detector from the vacuum chamber. The gas pressure in this cell can be varied in order to range out unwanted ions before they enter the ionization chamber. 


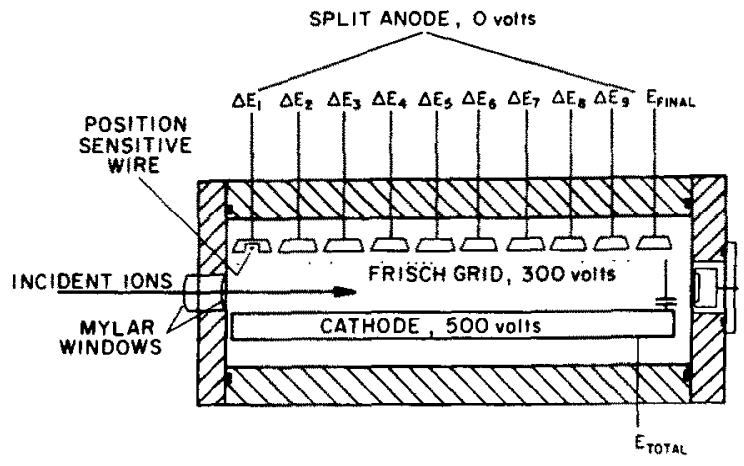

Fig. 4. A detailed view of the ionization chamber. This detection gives up to 10 measurements of energy loss in addition to a signal corresponding to the total energy deposited in the chamber.

ticles pass through a thin carbon foil. The first detector views a $2 \mu \mathrm{g} / \mathrm{cm}^{2}$ foil with a $1 \mathrm{~cm}$ aperture. This thin foil minimizes energy loss and multiple scattering. The second detector views a $5 \mu \mathrm{g} / \mathrm{cm}^{2}$ foil with a $2 \mathrm{~cm}$ aperture. The larger aperture accommodates the multiple scattering in the first time-of-flight foil. The overall time resolution obtained with this system is 400 ps.

After the time-of-flight system a multi-plate gas ionization detector measures differential energy loss, range and total energy. Fig. 4 shows a side view of this detector. Ionization electrons drift up through a Frisch grid and are collected on a split anode plate. The charge on each plate is a measure of the energy loss in a fixed region of the gas. The total energy is measured by the induced pulse on the cathode. This method has yielded a total energy resolution of $\pm 1 \mathrm{MeV}$ and allows for separation of neighbouring isobars.

\section{Mass independence}

The mass independence of the beam optics of the system was established by using samples of carbon and gold. Beams of these samples were analyzed by a $30^{\circ}$ magnet (resolution $4 \%$ ) and measured in the low energy Faraday cup. For each sample the mass region from 1 to $100 \mathrm{amu}$ was scanned and the relative yield $\mathrm{C}^{-}$/total source output and $\mathrm{Au}^{-} /$total source output measured. Samples of carbon and gold were then measured in the zero degree configuration. With the system tuned to maximize gold, $80 \%$ of the expected carbon was observed at the time-of-flight detector, establishing the mass independence of the beam line. The small mass dependence was attributed to residual fields in the magnetic elements which are not used in the electrostatic system. Since particles heavier than gold will be more rigid in magnetic fields, losses due to these stray fields will be no worse at higher masses.

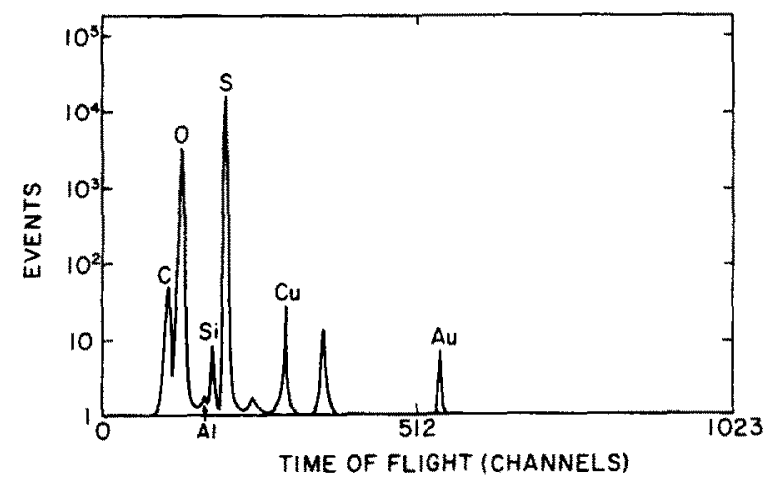

Fig. 5. A time-of-flight spectrum for a sample containing a mixture of beryllium, copper and gold. The other detected elements correspond to other materials present in the source and in the sample. The unlabeled peak between copper and gold corresponds to $S_{3}^{-}$molecular ions which strip to $\mathrm{S}^{+}$at the terminal.

Transmission losses through the accelerator are due primarily to the stripping of the negative ions in the residual gas in the low energy end of the accelerator. These losses have been measured by Lund et al. [12] to range from about $10 \%$ for $\mathrm{O}^{-}$up to $75 \%$ for $\mathrm{Au}^{-}$. Theoretical calculations of charge changing cross-sections indicate that they can be written as $\sigma=$ $f(Z) g\left(v / v^{\prime}\right)$, where $v^{\prime}=e^{2} / h=0.007 c$ is a velocity typical for the outermost electrons in an atom [13]. Data summarized by Allison and Garcia-Munoz [14] indicate that $g\left(v / v^{\prime}\right)$ has a broad maximum at $v / v^{\prime}=1$ and drops by about a factor of 10 at $v / v^{\prime}=10$, which is a characteristic velocity for light ions such as oxygen in

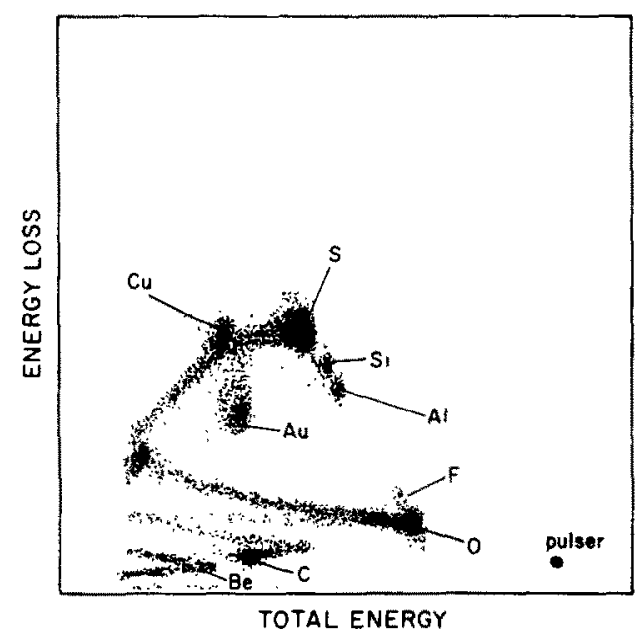

Fig. 6. A scatter plot of energy loss under the first plate of the ionization detector (vertical axis) and total energy deposited in the detector (horizontal axis). The horizontal and vertical axes have different scales. The peaks are identified by correlating them with their corresponding time-of-flight information. 
the low energy end of the accelerator. At $v / v^{\prime}=0.1$, $g\left(v / v^{\prime}\right)$ falls below its peak value by about a factor of 3 . This indicates that for any particular value of $Z$, the variation in stripping cross-section with mass is less than a factor of 10 . Thus, for low $Z$ ions such as oxygen, we expect a maximum variation in the transmission efficiency for heavy isotopes relative to normal ions to be about a factor of 3 .

These results were checked with a sample containing beryllium, copper and gold in a ratio measured using the $30^{\circ}$ magnet. Fig. 5 is a time-of-flight spectrum for this sample demonstrating that elements between beryllium and gold are simultaneously transmitted through the system. Some of the peaks arise from impurities in the sample and from the residual gas in the ion source. Fig. 6 shows a scatterplot of energy loss under the first plate versus total energy where the various elements have been identified using the time-of-flight system. Peaks corresponding to monochromatic energies are observed for elements from carbon to gold. The tails are caused by edge scattering from a $\mathrm{Ni}$ mesh attenuator which was introduced to limit the count rate of the beam in the detector. The lighter elements appear to be double-valued in energy loss since they escape from the detector. Elements from carbon to sulfur stop in the end of the detector. The heavier copper and gold samples range out under the first plate, so that for these ions, the energy loss equals the total energy.

\section{First experiments}

After the initial tuning of the system outlined above two test experiments were performed: a search for super heavy beryllium (>100 amu) and a search for heavy fractional charges in residual gases.

The negative ions used in the beryllium search were made by sputtering beryllium metal. With this source the yield of $\mathrm{Be}^{-}$ions is fairly low. Typical negative ion currents were $10 \mathrm{nA}$. The $\mathrm{Be}^{-}$ions were stripped to charge state $1+$ at the terminal of the accelerator at 5 $\mathrm{MeV}$ and required to remain in charge state $1+$ at the high energy stripper. This yielded an enhancement of heavy beryllium to ${ }^{9} \mathrm{Be}$ of $-10^{6}$. The electrostatic analyzer was set for an energy/charge ratio of $10 \mathrm{MV}$, corresponding to ions which strip to $1+$ at both strippers. The total rate of ${ }^{9} \mathrm{Be}$ observed at the time-offlight detector was $\sim 10^{6} / \mathrm{h}$. At the entrance of the ionization detector a gas absorber was used to eliminate ions with $Z$ greater than 6 . In a four hour run no events were observed with time-of-flight or energy loss signals consistent with masses greater than $100 \mathrm{amu}$.

In the search for fractional charges, the sample preparation was based on an idea of Schaad et al. [15] that any fractionally charged nuclei in nature would ultimately be captured into electrically neutral molecules.
These molecules are expected [15] to have binding energies similar to those of noble gases. The sample consisted of the gaseous residue of a xenon fractional distillation process (this sample was provided by Union Carbide Corp.). This purification process biases us toward masses greater than 80 amu and should result in an enrichment factor of about $10^{6}$. Typical beam currents were $1 \mu \mathrm{A}$. The electrostatic system was set to pass ions with initial charge $-\frac{1}{3}$ and charge $+\frac{2}{3}$ at the terminal, which was set to $6.67 \mathrm{MV}$. As in the beryllium run no events were observed in the time-of-flight or ionization detector consistent with masses greater than $80 \mathrm{amu}$ and consistent with non-integral charge.

\section{Results and conclusions}

The system described is ideally suited to search for high mass states of matter. In the search for an anomalously heavy isotope of beryllium a preliminary upper limit of $10^{-13}$ was deduced for the heavy hadron to nucleon abundance ratio. In the fractional charge search conducted with the residue of noble gases, a preliminary limit of $10^{-18}$ fractional charges per normal nucleus was achieved.

The limits presented here in our test run compare well with previous results [6-8] and are certainly well within the interesting range of expected effects [16].

We wish to thank D. Fisher, J. Fitch, T. Lund, K. Purser and G. Khalsa for their help in designing and assembling the system described. This work was supported in part by grants from the National Science Foundation and the Department of Energy.

\section{References}

[1] L.W. Jones, Rev. Mod. Phys. 49 (1977) 717.

[2] G.S. LaRue, J.D. Phillips and W.M. Fairbank, Phys. Rev. Lett. 46 (1981) 967; G.S. LaRue, W.M. Fairbank and J.D. Phillips, Phys. Rev. Lett. 42 (1979) 142; G.S. LaRue, W.M. Fairbank and A.F. Hebard, Phys. Rev. Lett. 38 (1977) 1011.

[3] D.C. Joyce et al., Phys. Rev. Lett. 51 (1983) 731.

[4] S.L. Glashow, Nucl. Phys. 22 (1961) 579; A. Salam and J.C. Ward, Phys. Lett. 13 (1964) 1681; S. Weinberg, Phys. Rev. Lett. 19 (1967) 1246.

[5] R. Cahn and S.L. Glashow, Science 213 (1981) 607.

[6] P.F. Smith et al., Nucl. Phys. B206 (1982) 333.

[7] J. Klein, R. Middleton and W.E. Stephens, Symp. on Accelerator Mass Spectrometry, Argonne National Lab (1981) p. 136.

[8] W.J. Dick et al., Phys. Rev. Lett. 53 (1984) 431.

[9] R. Middleton, Nucl. Instr. and Meth. 144 (1977) 373.

[10] I.S. Dmitriev and V.S. Nikolaev, Sov. Phys. JETP 20 (1965) 409. 
[11] J.D. Bowman and R.H. Heffner, Nucl. Instr. and Meth. 148 (1978) 503.

[12] T.S. Lund, Rev. Phys. Appl. 12 (1977) 1341.

[13] H.D. Betz, Rev. Mod. Phys. 44 (1972) 465.

[14] S.K. Allison and M. Garcia-Munoz, in: Atomic and Molecular Processes, ed., D.R. Bates (Academic Press, New York, 1962).
[15] L.J. Schaad et al., Phys. Rev. A23 (1981) 1600.

[16] S. Wolfram, Phys. Lett. 82B (1979) 65; C.B. Dover. T.K Gaisser and G. Steigmen, Phys. Rev. Lett. 42 (1979) 1117; R.N. Boyd, R.E. Turner, M. Wiescher and L. Rybarcyk, Phys. Rev. Lett. 51 (1983) 609. 